\title{
Posiciones en neurocirugía
}

\section{Neurosurgery positions}

Joel Marchant Kemp¹, Eduardo López Ferrada² ${ }^{2}$ Florencia Hermosilla Flores ${ }^{3}$

\begin{abstract}
Patient position is an integral part of operative planning. Pressure-related injuries can be prevented, the corridor expanded using gravity retraction, and surgeon fatigue minimized. Positioning the patient for surgery often does not receive the attention it deserves. The small openings leading into Deep operative fields require ergonomic patient positions to ensure a precise operative trajectory.
\end{abstract}

\section{RESUMEN}

La posición del paciente es una parte integral de la planificación operatoria. Se pueden prevenir las lesiones relacionadas con la presión, expandir el pasillo operatorio usando retracción por gravedad y minimizar la fatiga del cirujano. La colocación del paciente para la cirugía frecuentemente no recibe la atención que merece. Las pequeñas aberturas que llevan a los campos operatorios profundos requieren posiciones ergonómicas del paciente para asegurar una trayectoria operativa precisa.

\section{Key words:}

Neurosurgery,

positions, peripheral neuropathies, prone, supine

\author{
Palabras clave: \\ Neurocirugía, \\ posiciones, \\ neuropatías periféricas, \\ prono, \\ supino
}

\section{Introducción}

E I posicionamiento del paciente es un componente crítico de los procedimientos neuroquirúrgicos. Mientras que la mayoría de otras cirugías se realizan en la posición supina con poca participación o asistencia del anestesiólogo, el posicionamiento de los procedimientos neuroquirúrgicos requiere cooperación entre el cirujano, anestesiólogo y el personal de enfermería.

La colocación correcta del paciente es esencial para asegurar el adecuado acceso quirúrgico, como-

\footnotetext{
Anestesiólogo Hospital Guillermo Grant Benavente, Concepción. Profesor Asociado Facultad de Medicina Universidad de Concepción. Chile.

2 Neurocirujano Hospital Guillermo Grant Benavente. Concepción, Chile.

3 Anestesióloga Hospital Guillermo Grant Benavente. Concepción, Chile.
}

Fecha de ingreso: 22 de septiembre de 2020

Fecha de aceptación: 25 de noviembre de 2020

\section{ORCID}

0000-0002-5276-0066

Correspondencia:

Dr. Joel Marchant

joelmarchantkemp@gmail.com 
didad del cirujano, y un riesgo mínimo de lesiones en el paciente. Son variadas las posiciones empleadas comúnmente para procedimientos neuroquirúrgicos. Es esencial que todo el personal de la sala de operaciones tenga una comprensión global de las diversas posiciones empleadas para los procedimientos, así como los riesgos únicos y complicaciones postoperatorias asociadas a cada posición.

Las cuatro posiciones principales utilizadas para procedimientos neuroquirúrgicos incluyen: Posición supina, lateral, prono, y sentada.

Los pacientes deben ser cuidadosamente evaluados antes de la cirugía, y los beneficios de una posición particular, que se relacionan con el acceso quirúrgico y comodidad para el cirujano deben sopesarse frente a los riesgos específicos de esta.

Cada etapa de ubicar al paciente debe ser un esfuerzo cooperativo y debe ser realizado de una manera eficiente y segura. La comunicación es vital para reducir al mínimo el riesgo del paciente.

Los principios generales de la colocación del paciente se discuten extensamente en la literatura. En esta revisión, centraremos nuestra discusión sobre las consideraciones específicas que se refieren al posicionamiento de los pacientes para procedimientos neuroquirúrgicos.

Varios principios pueden ser cruciales para determinar la colocación apropiada del paciente. Por lo general, no hay un solo factor, sino más bien una combinación de factores que deciden la trayectoria operativa deseada.

La forma corporal del paciente y las preferencias del cirujano desempeñan papeles importantes en la elección final de la posición. El proceso de toma de decisiones para la elección de la posición apropiada es multifactorial y debe ser cuidadosamente planificada y compartida con todos los miembros del equipo del quirófano por lo que la distribución de la sala se pueda ajustar en consecuencia.

Idealmente, la lesión debe ser el punto más alto en el campo operatorio, pero con las excepciones de algunas lesiones superficiales, esto frecuentemente no es factible debido a la necesidad de tracción por gravedad para alcanzar lesiones profundas. Además, la superficie expuesta de cráneo y el perímetro de la craneotomía del paciente preferiblemente deben ser paralelas al suelo.

El torso debe estar fijado antes de la cabeza, porque fijación de la cabeza por el soporte de cráneo, se expone a cambios más significativos que la posición del torso. La elección del uso de un soporte de cráneo estático con pinchos versus otro implemento de soporte para la cabeza (almohada u herradura), será de elección del neurocirujano según su preferencia.

Una posición operativa apropiada del paciente requiere:

1- Permitir la trayectoria intradural menos obstruida hacia la lesión objetivo y reducir al mínimo el riesgo de lesiones a las estructuras cerebrovasculares normales.

2- Mantener al paciente los más cercano posible a la posición fisiológica del cuerpo para reducir al mínimo lesiones relacionada con la presión y lesiones de nervios periféricos.

3- Permiten al cirujano mantener una postura de cuerpo ergonómico para llevar a cabo maniobras de microcirugía de forma relajada con el fin de minimizar la fatiga del cirujano.

Si el paciente se coloca en una posición óptima, el cirujano tendrá la oportunidad de ampliar el corredor operativo a través de la movilización de forma segura del cerebro o cerebelo utilizando retracción por gravedad.

La extensión de la cabeza del paciente para movilizar el lóbulo frontal, alejándolo de la base del cráneo anterior durante una craneotomía pterional, es un ejemplo de este principio. Además, una posición óptima de la cabeza puede mejorar la visualización del campo operatorio profundo en la limpieza de sangre a través de la gravedad. La posición de la cabeza lateral utilizada durante una craneotomía suboccipital ilustra este punto.

Muchas posiciones de los pacientes tienen riesgos asociados únicos, los cuales deben ser apreciadas por el personal de enfermería, anestesiólogo y equipo quirúrgico.

El paciente debe ser evaluado de manera adecuada en el preoperatorio, y la posición óptima debe ser considerada y planificada en ese momento. El equipo de enfermería y anestesiólogo debe ser notificado tan pronto como sea posible acerca de la posición planeada para que puedan preparar todo el equipo necesario. El anestesiólogo debe ser capaz de prever complicaciones potenciales asociadas con la posición prevista y estar preparados para tratar en consecuencia.

Los principios básicos de la colocación del paciente en relación con el relleno adecuado y el posicionamiento de las extremidades deben estar basadas en la American Society of Anesthesiologists Práctica para la prevención de neuropatías periféricas perioperatorias (Tabla 1).

Los ojos del paciente deben ser cerrados y fijados después de la inducción de la anestesia general, y la lubricación del ojo deben ser considerados para pro- 


\section{Tabla 1. Síntesis de las recomendaciones establecidas para prevención de neuropatía periféricas perioperatorias}

I. Historia clínica y examen físico

Evaluar si paciente puede tolerar cómodamente la posición quirúrgica anticipada.

II. Estrategias de posicionamiento específicas para extremidad superior

- Abducción del brazo en supino no debe superar los $90^{\circ}$

- Pacientes en posición prono pueden tolerar la abducción del brazo mayor de $90^{\circ}$

\section{Paciente supino con el brazo en un apoya brazo}

La extremidad superior debe colocarse para disminuir la presión en el surco postcondilio del húmero (surco cubital). La supinación o la posición neutral del antebrazo facilitan esta acción

\section{Paciente supino con brazos al costado}

- El antebrazo debe estar en posición neutral

- La flexión de codo puede aumentar el riesgo de neuropatía ulnar, pero aún no hay consenso respecto al límite aceptable de grados de flexión durante el periodo perioperatorio

- Presión prolongada del nervio radial en el surco espiral del húmero debe ser evitada

- Extensión del codo más allá del rango de confort evaluado en el preoperatorio, puede estirar el nervio mediano

- Evaluaciones periódicas durante en perioperatorio aseguran la mantención de la posición deseada

III. Estrategias de posicionamiento específicas para extremidad inferior

- Estiramiento del grupo muscular de los isquiotibiales: Posiciones que producen estiramiento mayor al rango de confort evaluado en el preoperatorio, puede producir estiramiento del nervio ciático

- Limitar flexión de la cadera: Dado que el nervio ciático y sus ramas cruzan tanto la articulación de la cadera y rodilla, la extensión y la flexión de estas articulaciones, respectivamente, deben ser consideradas al determinar el grado de flexión de la cadera en la posición quirúrgica

- Ni la extensión o la flexión de la cadera aumentan el riesgo de neuropatía femoral

- Presión prolongada sobre el nervio peroneo a nivel de la cabeza del peroné debe ser evitada

IV. Protección acolchada

- Tablas de brazo acolchadas: Disminuyen riesgo de neuropatía de extremidad superior

- Rollos pectorales: Su uso en posición lateral izada puede disminuir el riesgo de neuropatía de extremidad superior

- Protección acolchada en los codos: Disminuye el riesgo de neuropatía de extremidad superior

- Protección acolchada para el nervio peroneo: Su uso específico para prevenir la presión de estructuras duras sobre el nervio peroneo en la cabeza del peroné, puede disminuir el riesgo de neuropatía

- Complicaciones del uso de protecciones acolchadas: El uso inapropiado (ej: fijar con mucha presión) puede aumentar el riesgo perioperatorio de neuropatía

V. Equipamiento

- El uso de manguitos de presión arterial automatizados bien posicionados no cambia el riesgo de neuropatía de extremidad superior

- El uso de aparatos ortopédicos para el hombro en posición con cabeza inclinada, puede aumentar el riesgo de neuropatías perioperatorias

\section{Evaluación postoperatoria}

- Una evaluación postoperatoria simplificada del funcionamiento de los nervios de extremidades, pueden llevar a un reconocimiento precoz de neuropatía periférica

Resumen de recomendaciones tomado de: Practice Advisory for the prevention of perioperative peripheral neuropathies: an updated report by the American Society of Anesthesiologists Task Force on prevention of perioperative peripheral neuropathies, Anesthesiology 114:741-754, 2011

cedimientos largos. Los brazos se deben mantener en una posición neutral, con la abducción del brazo limitado hasta 90 grados. Las prominencias óseas de las extremidades deben estar acolchadas bien para evitar la compresión y las ulceras por presión de la piel, así como neuropatías periféricas.
Durante un breve período, los pacientes pueden ser desconectados de los dispositivos de vigilancia, líneas vasculares, y circuito de ventilación. Esto requiere una cuidadosa coordinación entre el cirujano y el equipo de anestesia para garantizar la seguridad del paciente, y el paciente debe ser reconectado al venti- 
lador y monitores de manera oportuna.

El posicionamiento del paciente es un desafío, requiere una adecuada profundidad anestésica, la mantención de la estabilidad hemodinámica, una apropiada oxigenación y la preservación de la monitorización invasiva. La desconexión de líneas arteriales, venosas y tubo endotraqueal es comúnmente necesario para realizar rotación o movilización del paciente, creando un completo "blackout" durante el cual el paciente no es monitorizado ni oxigenado.

\section{Posicionamiento de la cabeza}

La posición ideal de la cabeza es aquella que proporciona un enfoque quirúrgico óptimo para el área objetivo con una intrusión mínima del cerebro normal.

Es esencial el conocimiento de los abordajes quirúrgicos para evaluar la tolerancia del paciente a la posición deseada durante largos periodos de tiempo.

\section{Se basa en dos principios}

1- La trayectoria imaginaria desde el punto más alto de la superficie del cráneo hasta el área de interés en el cerebro debe ser la distancia más corta posible.

2- Siempre que sea posible, la superficie expuesta del cráneo y un perímetro imaginario de craneotomía deben ser paralelos al suelo.

\section{Alineación de la cabeza y el cuello}

\section{a. Rotación de la cabeza}

Es importante recordar que el cuello está alineado con la cabeza y el cuerpo. La cabeza puede rotarse con seguridad entre cero y $45^{\circ}$ lateralmente. Si se requieren más de 45 grados de rotación de la cabeza, el hombro ipsilateral se levanta sobre una almohada o rollo para mantener el eje[1]. La rotación extrema de la cabeza, en ocasiones puede ser extremadamente perjudicial. Tiene efecto sobre las estructuras vasculares del cuello. Disminuye el flujo sanguíneo en las arterias vertebrales ipsilaterales a medida que atraviesan los agujeros estrechos en el proceso transversal a lo largo de la columna cervical. También altera el retorno venoso de las venas yugulares internas, lo que lleva a un aumento de la PIC y la inflamación del cerebro, lo que aumenta el sangrado. Un metaanálisis evidencia que el flujo se reduce con mayor frecuencia en el lado contralateral de la rotación y más en la parte intracraneal en comparación a la parte cervical[2]. Se ha descrito la compresión mecánica de la arteria vertebral extracraneal durante la rotación del cueIlo[3]. Los pacientes con factores de riesgo asociados, como la espondilosis cervical, vértigo, aterosclerosis, osteoartritis, adultos mayores, etc. tienen más probabilidades de tener compromiso del flujo sanguíneo vertebral con la rotación lateral del cuello. Preoperatoriamente, la identificación de pacientes con signos de posible insuficiencia vertebro basilar (IVB), permitirá a los neurocirujanos y anestesiólogos posicionar de manera óptima el cuello. En tales individuos, la evaluación de la IVB con un Doppler USG transcraneal es beneficiosa.

\section{b. Flexión y extensión de la cabeza}

La hiperflexión de la cabeza también conduce a una disminución del flujo sanguíneo tanto en las arterias vertebrales como en las carótidas, lo que puede conducir a isquemia del tronco cerebral y de la columna cervical. También reduce el diámetro anteroposterior de la hipofaringe causando isquemia de la base de la lengua que conduce a edema faríngeo y de lengua. Se recomienda que distancia tiromentoniana no debe ser menor a 2 o 3 traveses de dedos[4]. La hiperextensión de la cabeza puede producir desplazamiento del tubo endotraqueal. La presión externa en el cuello debido a la fijación del tubo endotraqueal, también impiden el drenaje venoso, lo que resulta en condiciones quirúrgicas deficientes. Cualquier distensión evidente de la vena del cuello es un signo de una posición inadecuada de la cabeza y el cuello que contribuirá a elevar la PIC.

\section{c. Elevación de la cabeza}

La cabeza a menudo se coloca sobre el corazón para facilitar el retorno venoso. En pacientes en posición supina, $10^{\circ}$ de Trendelenburg inverso produce una disminución significativa en la PIC, mientras que la presión de perfusión cerebral (PPC) no se modifica[5]. Esto ocurre 1 minuto después del cambio de posición y permanece estable. Esto sucede también en la posición prona[6]. Según la doctrina de MunroeKelly, la disminución de la PIC debida a la posición inversa de Trendelenburg se debe al desplazamiento del líquido cefalorraquídeo (LCR) hacia el segmento espinal[7]. En pacientes en posición prona, los aumentos en la presión intraabdominal a menudo contribuyen al aumento de la PIC y, por lo tanto, elevar el extremo de la cabeza es vital para reducir la PIC.

\section{d. Monitoreo durante el posicionamiento de cabeza y cuello}

Aunque en la práctica no se utiliza un monitoreo de rutina adicional durante la colocación de la cabeza 
y el cuello, en pacientes vulnerables, el monitoreo de la PIC puede ser útil para alcanzar un objetivo de 20 $\mathrm{mmHg}$. La presión del bulbo yugular y la saturación venosa yugular son sustitutos de la monitorización de la PIC. Se puede controlar fácilmente con un catéter yugular retrógrado. La medición simultánea de la presión venosa central (PVC) y la presión venosa yugular (PVJ) con los puntos de referencia apropiados (PVC en la aurícula derecha y PVJ al nivel de tragus) no debe mostrar cambios en las presiones en comparación con la línea de base con un posicionamiento óptimo. Se debe considerar la obstrucción parcial de la salida venosa en caso de que haya un aumento en la PVJ, y se debe realizar un rápido reposicionamiento de la cabeza[8].

\section{e. Fijación de la cabeza para craneotomía}

La fijación de la cabeza después de decidir la posición final de la cabeza es un paso crucial en la colocación del procedimiento neuroquirúrgico. La cabeza puede colocarse en una variedad de dispositivos de fijación dependiendo de la cirugía. Los dispositivos comunes que se utilizan son la abrazadera de cabeza Mayfield Kess, el armazón de cabeza Sugita y el reposacabezas tipo herradura (Figura 1) (Tabla 3).

Cabezal Mayfield: Consiste en una pinza craneal con tres pernos de fijación estériles. Se debe tener especial cuidado en evitar el seno frontal, el hueso temporal y los senos venosos. No debe interferir con la incisión craneal. Cuando las pinzas se aprietan juntas, los pernos se deslizan hasta que las puntas están asentadas en el cráneo. Cada anillo del perno ejerce una presión de $20 \mathrm{lb}$. En los adultos, se permiten hasta 80 lb de presión, mientras que, en la población pediátrica, el límite preferible es de 30 a 40 lb. Luego se sujeta al ensamblaje del marco de la cabeza que se adjunta a la mesa (Figura 1). El fabricante recomienda el uso de clavijas pediátricas especialmente diseñadas para niños menores de 10 años, pero es preferible evitar el uso de Mayfield en niños menores de 3 años[9].

Marco de cabeza Sugita: Diseñado por el Dr. Sugita utiliza cuatro pernos de fijación para colocar la cabeza del paciente (Figura 1). Es ideal para procedimientos donde se necesita el máximo soporte. Permite una rotación de $360^{\circ}$ del ángulo de la cabeza durante la cirugía. Se compone de un conjunto de marco de cabeza robusta y un soporte de cabeza. Los retractores automáticos se unen a los marcos de la base utilizando tornillos de mariposa que están espaciados en ángulos de $35^{\circ}$. La barra semicircular montada en el marco basal se utiliza para sujetar los ganchos del cuero cabelludo o los retractores automáticos.

Reposacabeza tipo herradura: Consiste en un reposacabezas en forma de herradura con ajustes tanto verticales como laterales que proporcionan flexibilidad en la posición del paciente tanto en posición supina como en posición prona. También tiene almohadillas de gel que lo hacen cómodo para su uso. La polea para la tracción esquelética lo convierte en una opción atractiva para los procedimientos de columna cervical. Se deben tomar precauciones para evitar la presión sobre la cara en las posiciones prono que pueden provocar necrosis de la frente y lesiones en los ojos. La alopecia del cuero cabelludo es una complicación conocida en pacientes colocados en posición

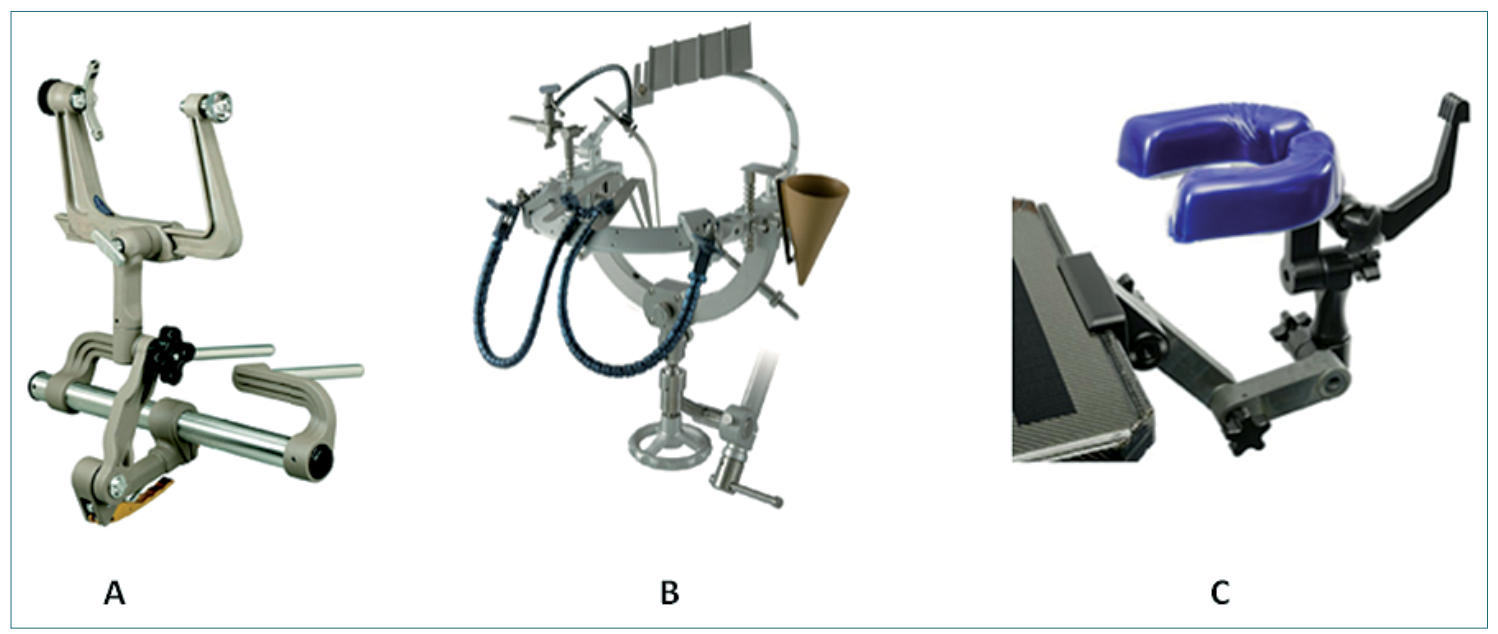

Figura 1. Dispositivos de fijación de la cabeza. A: Cabezal Mayfield; B: Marco de cabeza Sugita; C: Reposacaveza tipo herradura. 
Tabla 2

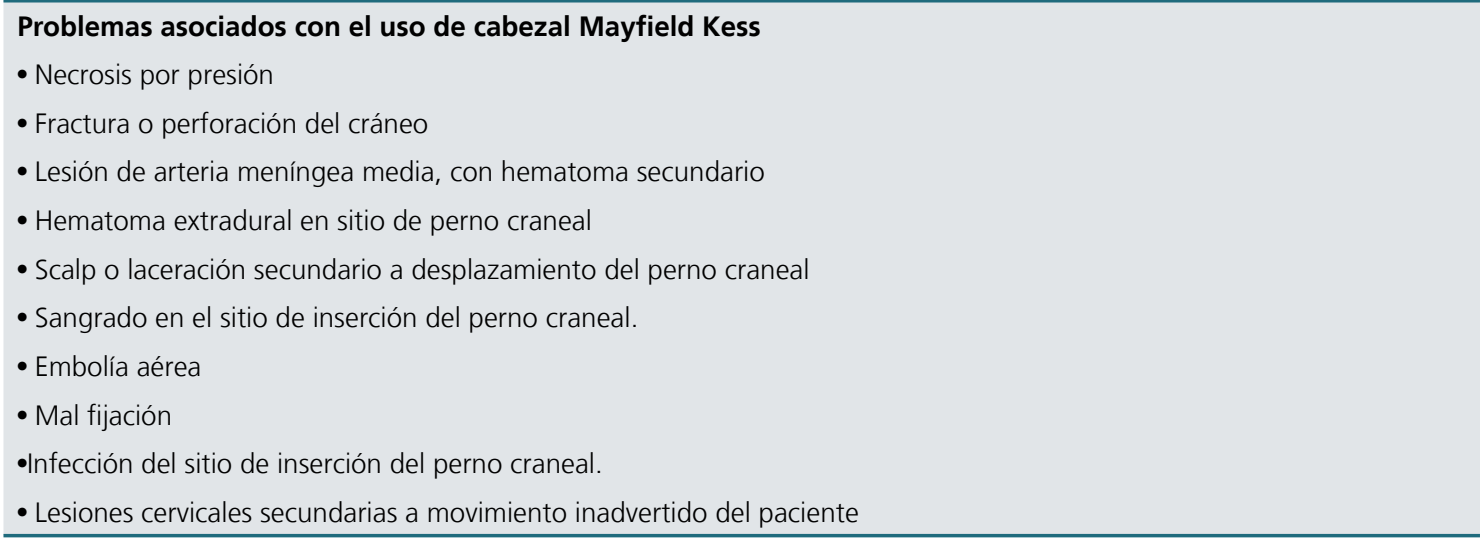

supina en el reposacabezas de herradura por una duración prolongada.

\section{Posicionamiento del cuerpo}

\section{a) Posición supina o decúbito dorsal}

Es la posición más utilizada en neurocirugía. No requiere ningún equipo especial para el posicionamiento y, a menudo, se puede lograr fácilmente porque el paciente puede moverse a la cama por sí mismo, lo que permite que la mayoría del posicionamiento se complete antes de la inducción de la anestesia. Es seguro ya que no requiere la desconexión del tubo endotraqueal ni otros dispositivos de monitoreo.

En esta posición, el volumen sistólico (VS), el gasto cardíaco (GC) y el retorno venoso son óptimos, y hay una disminución mínima de la presión arterial media. La capacidad residual funcional (CRF) y el volumen pulmonar total (VPT) disminuyen debido a la atelectasia de las zonas pulmonares dependientes, lo que causa desajuste entre la ventilación y la perfusión (V/Q). Se ha demostrado que los niveles de $\mathrm{PaCO}_{2}$, 30 minutos después de la inducción anestésica y la posición supina, se elevan debido al aumento del espacio muerto alveolar[10]. La PPC se mantiene pero el drenaje del LCR puede verse afectado. La cabeza debe colocarse por encima del nivel del corazón para promover el drenaje venoso y reducir el edema cerebral. La cabeza puede girarse hasta $45^{\circ}$ con respecto al cuerpo, pero si se necesita más rotación, se debe colocar un rollo o almohada debajo del hombro contralateral. Esto también causa un leve desplazamiento de las vísceras abdominales hacia abajo, mejorando la ventilación.

Después de la colocación final de la cabeza, ya sea en la posición de flexión o extensión, es importante descartar la migración endobronquial del tubo endotraqueal. Es preferible fijar el tubo endotraqueal en el lado opuesto a la cirugía para evitar desconexiones accidentales. Ciertos procedimientos, como el abordaje transoral del odontoide, requieren la colocación del tubo en el centro de los labios. Es importante evitar el contacto piel a metal. El brazo debe colocarse de manera que se minimice la presión en la ranura cubital. La posición neutral del antebrazo se debe mantener cuando los brazos estén hacia adentro. Debe haber un acolchado adecuado en el nervio cubital para evitar la neuropatía cubital. La lesión del plexo braquial puede ocurrir si los brazos se abducen más de $90^{\circ}$, donde la cabeza del húmero actúa como un punto de apoyo alrededor del cual se estiran los nervios del plexo braquial[11]. La presión prolongada sobre el nervio peroneo en la cabeza del peroné debe minimizarse con un protector acolchado. Los puntos de contacto óseo en el codo, rodilla, hueso occipital, sacro y talón deben estar acolchados[12]. El paciente debe estar firmemente sujeto a la mesa de operaciones por correas de seguridad, que posicionan través del torso y muslo del paciente para que los vasos no se obstruyan. La posición supina clásica conduce a la pérdida de lordosis lumbar y puede causar dolor de espalda postoperatorio.

La posición supina a menudo se modifica ligeramente en una posición contorneada (posición de silla de jardín) o posición de Trendelenburg inversa. La silla de jardín o la posición contorneada (Figura 2) es fisiológicamente más favorable para la columna lumbosacra. Se proporciona una angulación y flexión de $15^{\circ}$ en el tronco, el muslo y la rodilla. La rodilla se mantiene flexionada por una almohada debajo de ella. Esta posición también está asociada con la mejora del 


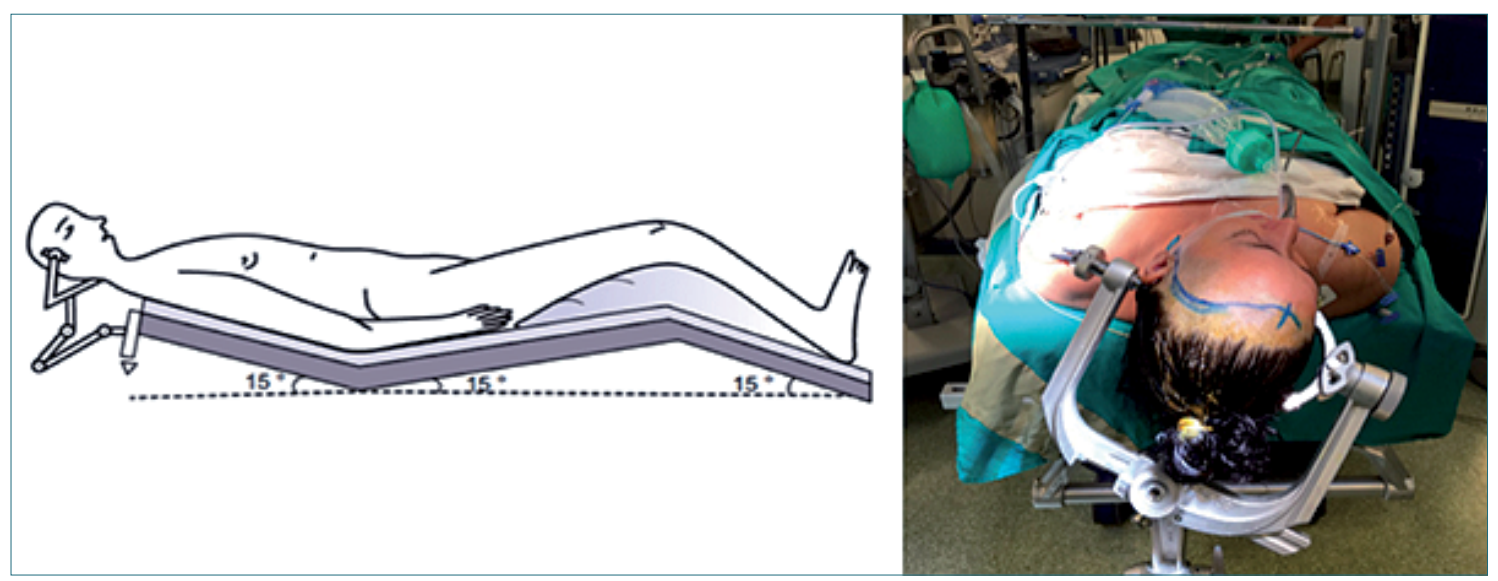

Figura 2. Posición supino. A: Posición en silla de jardín; B: Imagen de paciente en decúbito supino con cabezal mayfield, cabeza ligeramente rotada para abordaje pterional. Imagen tomada de: G. Singh. Christian Medical College, Vellore, India. POSITIONING IN NEUROSURGERY. 2017 Elsevier.

retorno venoso desde las extremidades inferiores con un drenaje de LCR y linfático óptimos.

La posición de inclinación hacia arriba o hacia atrás de Trendelenburg implica una inclinación de 10 a $15^{\circ}$ respecto al eje horizontal, y mejora el drenaje venoso desde el corazón.

\section{b) Posición lateral}

En la posición lateral, los parámetros hemodinámicos se modifican mínimamente con una leve disminución del VS, GC y un aumento de la resistencia vascular sistémica (RVS) y la resistencia vascular pulmonar (RVP). Esto conduce a una disminución moderada de la presión arterial sistólica y la presión arterial media (PAM) en comparación con la posición supina. Con respecto a la ventilación, hay un aumento leve en la $\mathrm{PaO}_{2}$ en comparación con la posición supina[10]. La perfusión es mejor en la zona pulmonar dependiente, mientras que el pulmón no dependiente está mejor ventilado, lo que causa una leve falta acoplamiento V/Q. Sin embargo, dado que el abdomen está libre en comparación con la posición supina, su impacto en la oxigenación es limitado.

Con la flexión extrema del cuello, existe la posibilidad de disminución del flujo venoso yugular (FVY), con aumento resistencia venosa yugular (RVY) y la PIC. Se coloca un rodillo axilar debajo del tórax debajo de la axila para evitar la compresión axilar. Cuando la cabeza está en el Mayfield, el hombro dependiente se lleva más allá del borde cefálico de la mesa del quirófano y el brazo dependiente se apoya en un reposabrazos acolchado bajo que se inserta entre la mesa y la fijación de la cabeza. En estos casos, no hay ne- cesidad de un rodillo axilar. El brazo no dependiente generalmente se coloca sobre el tronco en una almohada. Para controlar el flujo de sangre al brazo dependiente, es prudente colocar la sonda de saturación o la línea arterial en el brazo dependiente (Figura 3). El tronco se apoya sobre apoyos laterales y se sujeta mediante cintas. Cuando la cabeza no está fijada en MFK, es importante apoyar la cabeza del paciente con una almohada para evitar la angulación de la columna cervical. Las extremidades inferiores se colocan con una almohada entre las piernas y las extremidades dependientes se flexionan para evitar la presión sobre la cabeza del peroné y el nervio peroneo. Las compli-

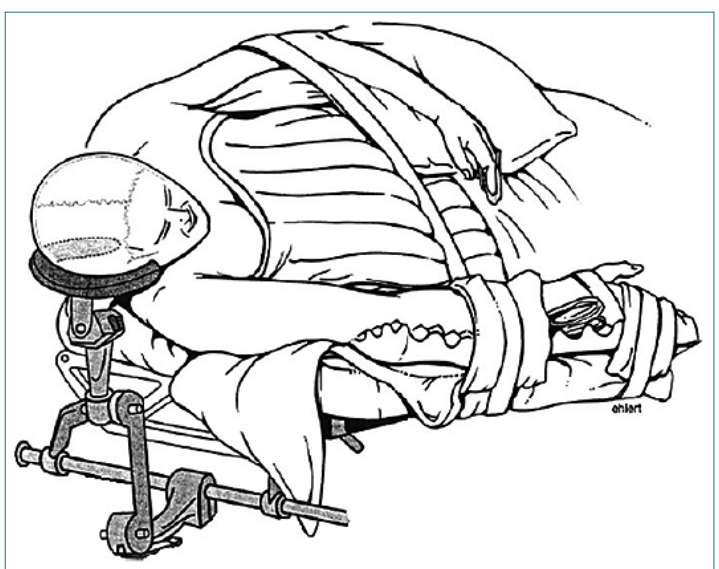

Figura 3. Posición lateral. Imagen tomada de: Rozet I, Vavilala MS. Risks and benefits of patient positioning during neurosurgical care. Anesthesiol Clin September 2007;25(3):63153. 
caciones que se anticipan con la posición lateral son la lesión del plexo braquial, el compromiso vascular de la extremidad superior dependiente, las lesiones del oído y los ojos y la lesión del nervio supraescapular del hombro dependiente.

\section{c) Posición del banco del parque "Park Bench"}

Es una modificación de la posición lateral, proporciona un mejor acceso a las lesiones craneales bajas y proporciona al cirujano acceso al tronco cerebral anterior y al foramen magnum, así como a los tumores de ángulo cerebelopontino. En esta posición, el tronco se gira $15^{\circ}$ desde la posición lateral. La parte superior del brazo se coloca a lo largo del tronco lateral y la parte superior del hombro se sujeta con cinta adhesiva hacia la mesa. El hombro y el brazo dependientes se colocan fuera de la cama de quirófano y el brazo se apoya en un cabestrillo. La extremidad inferior está ligeramente flexionada y se coloca una almohada entre las rodillas. Es importante no pegar el hombro con cinta adhesiva o dejar caer demasiado el cuello (Figura 4).

\section{d) Posición Semilateral (Posición Janetta)}

Esta posición lleva el nombre del neurocirujano que popularizó su uso para la descompresión microvascular del quinto nervio craneal. Se logra mediante la inclinación semilateral de la mesa combinada con un generoso balanceo de hombros. Debe evitarse la rotación extrema de la cabeza que puede causar la compresión de la yugular contralateral por la barbilla. La cabeza se gira ligeramente hacia afuera del lado afectado y se flexiona aproximadamente a dos dedos del esternón. El vértice de la cabeza se mantiene paralelo al piso (Figura 5).

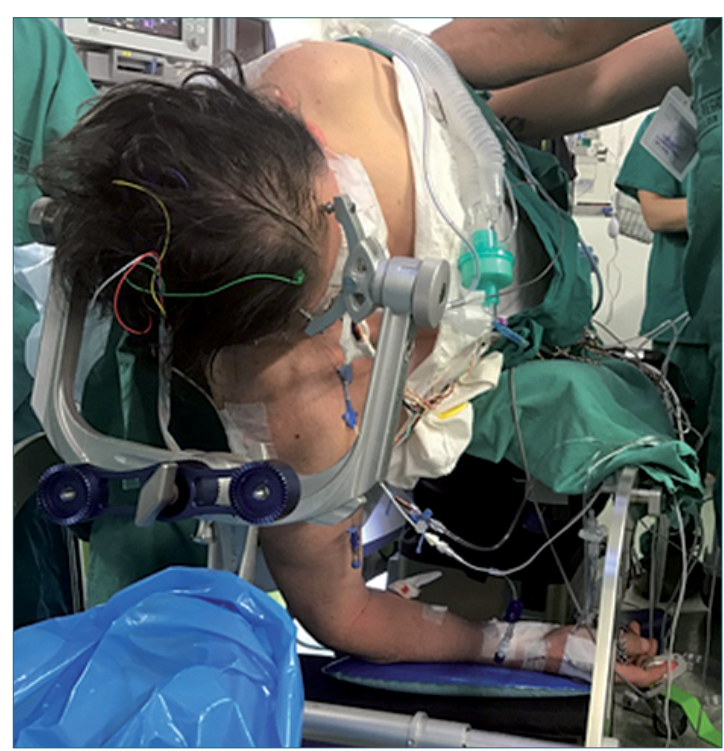

Figura 4. Posición park benck: Brazo inferior con apoyabrazos, brazo superior descansa sobre cuerpo de paciente, con cables de dispositivos para monitoreo neurofisiológico. Posición de abordaje retrosigmoideo para tumor del ángulo pontocerebeloso.

\section{e) Posición prono}

En la posición prono, hay importantes cambios cardiovasculares y respiratorios. El retorno venoso y el VS se reducen significativamente debido al aumento de la presión intraabdominal y la acumulación de sangre en las extremidades que conducen a una disminución del GC, una disminución de la fracción de eyección del ventrículo izquierdo y del índice cardíaco (IC). Para compensar la reducción significativa del GC,
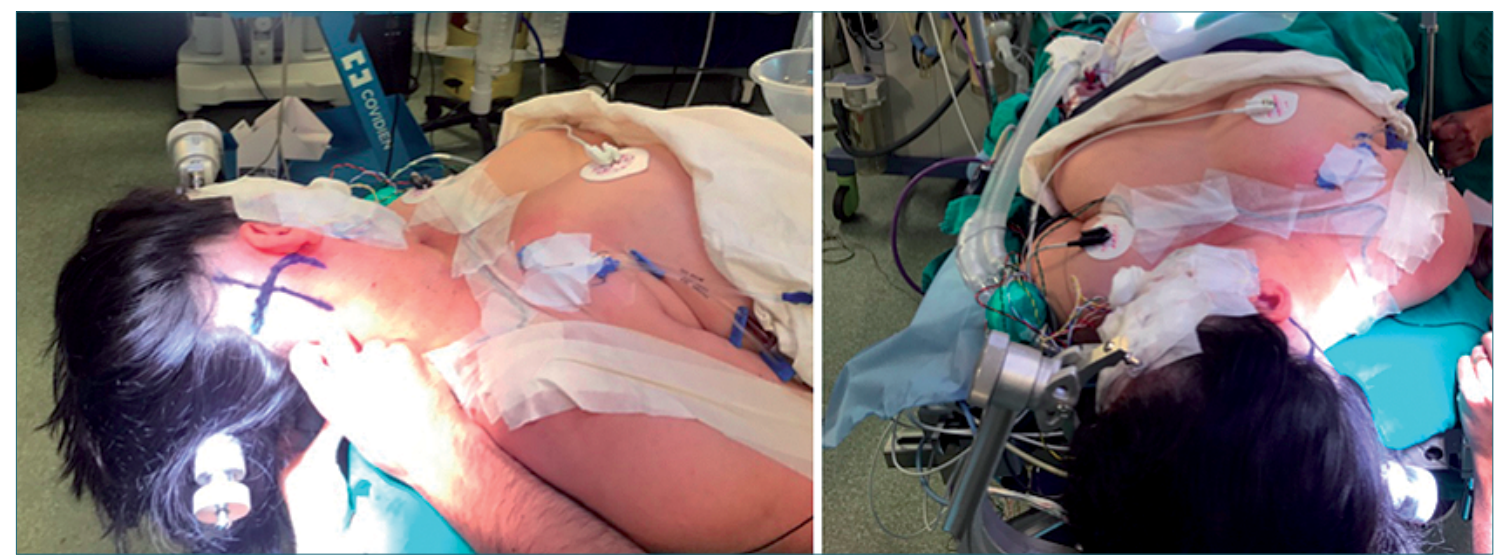

Figura 5. Posicion Janetta: Posición de abordaje retrosigmoideo para descompresión neurovascular. 
la frecuencia cardíaca aumenta junto con los aumentos en RVS y RVP.

En comparación con la posición supina, el CRF y el VPT aumentan y hay una perfusión mejorada. Además, hay una disminución en la atelectasia debido a la apertura de la zona dorsal de los pulmones, lo que minimiza el trastorno $\mathrm{V} / \mathrm{Q}$, lo que mejora la oxigenación. Aunque hay una disminución en la compliance de la pared torácica debido al aumento de la presión intraabdominal, esto generalmente se supera con la ventilación con presión positiva. En general, la oxigenación mejora con la posición prona[10].

La hemodinámica cerebral varía con la posición de la cabeza. Cuando la cabeza se mantiene neutral, la FVY aumenta y la RVY disminuye. Sin embargo, si la cabeza es más baja que el corazón, hay congestión venosa que lleva a un aumento de la PIC[8].

Para posicionarse en posición prona, el paciente suele ser anestesiado en la camilla y luego rotado hacia la mesa de operaciones, que se prepara con rollos de tórax colocados en forma longitudinal / transversal. La cabeza se fija al dispositivo de fijación de la cabeza, antes de rotar al paciente. Existen múltiples problemas potenciales con el giro del paciente, como la desconexión de monitores, accesos IV, tubo orotraqueal, etc. Todo esto puede contribuir a un retraso en el reconocimiento de incidentes anestésicos como hipotensión, hipoventilación, desaturación, arritmias o paro cardíaco. Se requiere una vigilancia extrema durante el giro del paciente, y es prudente mantener el oxímetro de pulso y una línea invasiva/ECG para el reconocimiento temprano de estos eventos. Todos los catéteres [urinarios, venosos centrales, drenaje toráci$\mathrm{CO}$, drenaje ventricular externo (DVE)] deben asegurarse firmemente al cuerpo antes de girar.

Una vez que el paciente se encuentra boca abajo, la barbilla del paciente debe estar libre de la mesa. La flexión, rotación o extensión extremas del cuello pueden perjudicar el retorno venoso y, por lo tanto, aumentar la PIC. Los puntos de presión, como las rodillas, las ingles, deben estar acolchados. Los senos deben estar desplazados medialmente y debe evitarse la presión sobre los pezones. Se deben revisar los pulsos periféricos de la extremidad inferior para descartar el compromiso vascular de los vasos femorales. Las extremidades superiores se colocan al lado del paciente con las manos hacia el paciente. Los ojos, la nariz, las orejas deben estar protegidos y los párpados deben estar cerrados. Es importante asegurar firmemente el tubo endotraqueal para evitar la extubación accidental en la posición prona. Se coloca un rollo de gasa suave como un bloque de mordida para evitar la mordida involuntaria de la lengua durante el monito- reo de los potenciales evocados por el motor y para mantener de manera segura el tubo endotraqueal en su lugar.

También se puede considerar la intubación orotraqueal reforzada para evitar el retorcimiento. El anestesiólogo debe estar preparado para una pérdida repentina en la vía aérea en la posición prona. Se han descrito informes sobre manejo de vía aérea con máscara laríngea en posición prona como una alternativa de emergencia a la intubación endotraqueal[13]. Dado que la posición prona se ha asociado con cambios hemodinámicos significativos, es prudente precargar al paciente adecuadamente antes de girar y también estar preparado para manejar la inestabilidad hemodinámica repentina en la posición prona. Los pacientes que son susceptibles a un compromiso hemodinámico significativo, como aquellos con diabetes no controlada o hipertensión, pueden requerir un tratamiento con vasopresores mientras se prona[14]. Deben hacerse planes de respaldo para un paro cardíaco repentino en pacientes con comorbilidades significativas.

\section{Posición prona en cirugía de columna: (Figura 6) Tabla 2}

Complaince pulmonar: Al comprimir el abdomen y limitar el movimiento de la pared torácica, existe un compromiso significativo de la complaince pulmonar en la posición prona. En un estudio que comparó los tres dispositivos de posicionamiento de uso común, los rollos de tórax colocados longitudinalmente, la tabla de la columna vertebral de Jackson y el marco de Wilson, han demostrado que los pacientes que se someten a procedimientos en la posición prona tienen un aumento en la presión máxima de las vías respiratorias y una disminución de la distensibilidad pulmonar cuando se mueven de la posición supina a la prona tanto en el grupo con rollos de tórax como en el marco de Wilson, pero no en la mesa de Jackson lo que sugiere que los mecanismos pulmonares dependen del dispositivo usado[26]. La libre circulación del abdomen mejora el desplazamiento del diafragma, mejorando así la oxigenación y la ventilación. También disminuye el sangrado al disminuir la presión intrabdominal que favorece el retorno venoso desde las extremidades inferiores (Figura 6).

Factores relacionados con la posición que afectan la pérdida de sangre en procedimientos espinales en posición prono: Las venas vertebrales están conectadas con las que están en el tórax a través del canal vertebral y las que están en el abdomen y la pelvis a través de las venas intercostales, lumbares 


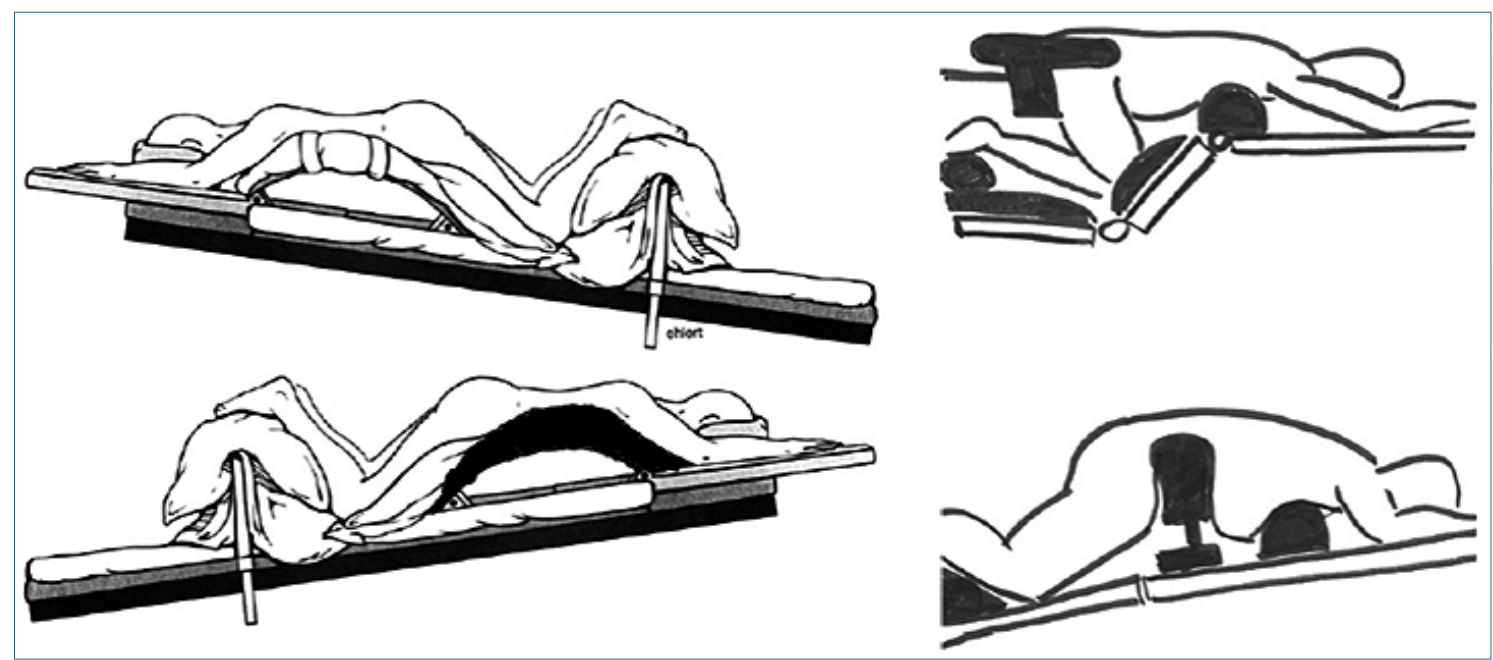

Figura 6. Ejemplos de posición prono en cirugía de columna. Imagen tomada de: Rozet I, Vavilala MS. Risks and benefits of patient positioning during neurosurgical care. Anesthesiol Clin September 2007;25(3):631-53.

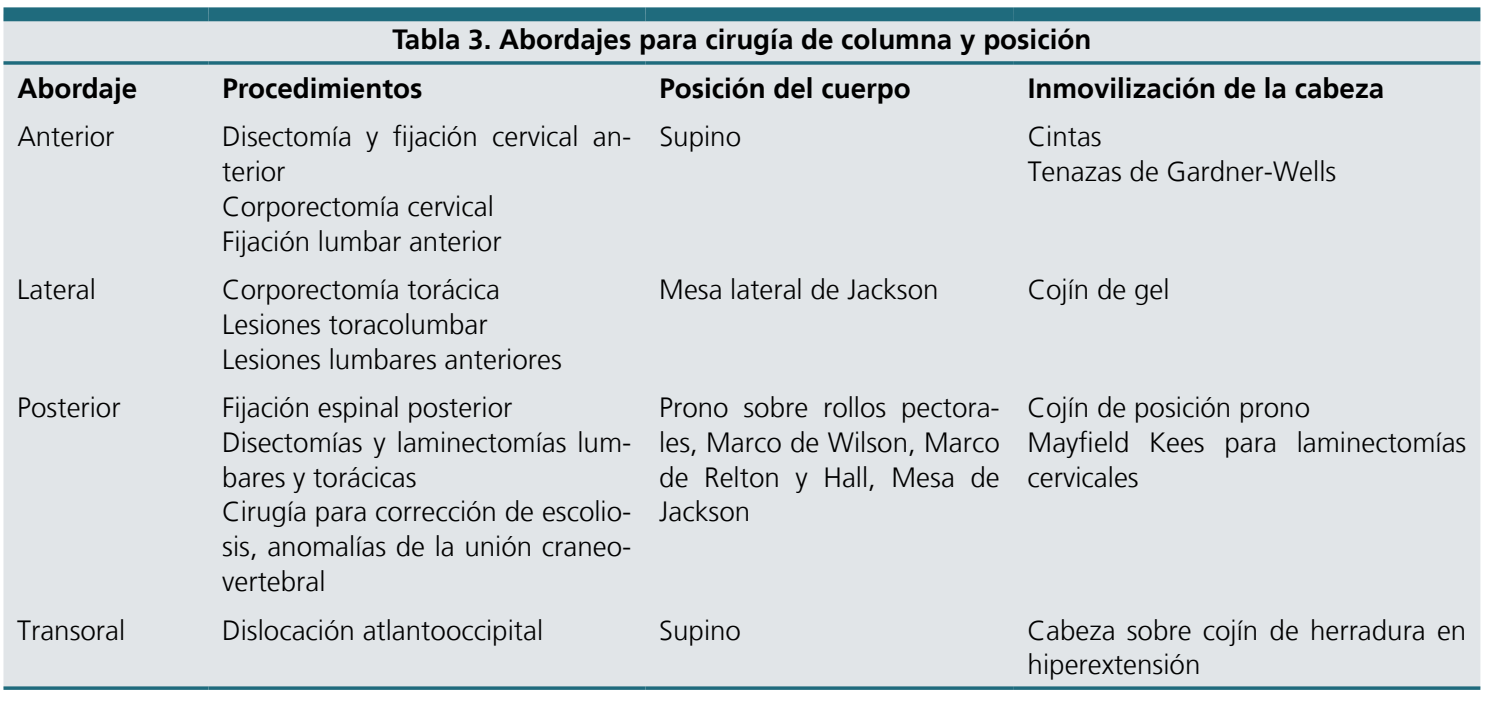

y otras. Con la obstrucción del retorno venoso, la sangre de la parte inferior del cuerpo podría desviarse en el sistema venoso vertebral.

\section{El plexo de Batson consiste en lo siguiente:}

1. Sistema venoso interno, que es continuo desde la base del cráneo hasta la región sacrococcígea. Está formado por las venas anteriores internas (en la superficie posterior del cuerpo vertebral), las venas internas posteriores (en la superficie de la lámina) y las venas anastomóticas entre las dos.

2. Sistema venoso externo que consiste en las venas transversas longitudinales anteriores a los cuerpos vertebrales en la cara externa de la lámina.

3. Venas anastomóticas o conectivas que son un grupo de venas que conectan el sistema venoso interno al externo y los sistemas venosos sistémicos y vertebrales.

Durante la posición prona, múltiples razones son responsables de la obstrucción del retorno venoso, esto provoca el desvío de la sangre hacia las venas vertebrales. La presión en la pared abdominal anterior debido a bolsas de arena, refuerzos o tensión 
muscular abdominal excesiva produce presión en la vena cava inferior (VCI)[27]. Incluso se sabe que un aumento moderado de la presión abdominal causa un aumento de la presión de la VCl. Alteración de la compliance pulmonar asociado con la posición prona puede causar presiones elevadas en las vías respiratorias que dificultan el retorno venoso al corazón, lo que disminuye el GC y aumenta la presión venosa sistémica. Esto a menudo también conduce a una disminución de la presión de perfusión de la médula espinal $(P P M E=P A M-$ presión venosa de la columna vertebral), lo que conduce a un potencial de aumento de las complicaciones neurológicas. La hipercarbia con aumento de la presión de la vía aérea durante la espiración también provoca un aumento de la presión venosa. Todos estos factores contribuyen a la pérdida de sangre en la posición prona. El posicionamiento óptimo es, por lo tanto, esencial para minimizar la pérdida de sangre en esta posición.

Alineación de la columna vertebral para los procedimientos de la columna vertebral: Es importante lograr una alineación correcta de la columna durante el posicionamiento para obtener el mejor resultado clínico. Cuando no se realiza la artrodesis, el objetivo en el posicionamiento intraoperatorio es proporcionar una descompresión neural óptima sin sangrado excesivo. Pero si se planea la artrodesis, el posicionamiento debe incluir la colocación de la columna vertebral en la alineación anatómica para evitar la deformidad iatrogénica. La alineación incorrecta de la región occipitocervical puede tener varias complicaciones. En la posición extremadamente extendida, la fusión de la columna vertebral conduce a la incapacidad del paciente para ver su propio cuerpo, mientras que, en la flexión excesiva, existe dificultad para tragar. La mala alineación rotacional conducirá a la inclinación de la cabeza. En la artrodesis lumbar, mantener una adecuada lordosis lumbar es crucial para prevenir el síndrome de espalda plana y, por lo tanto, es preferible evitar el marco de Wilson para los procedimientos de fusión de la columna lumbar[28].

Posicionamiento de la cabeza para los procedimientos de la columna vertebral: En casos de columna cervical inestable, la cabeza debe estabilizarse con un dispositivo de inmovilización rígido como el collar Filadelfia o la tracción cervical. Se debe realizar una transferencia cuidadosa y meticulosa de la posición supina a la posición prona mientras se mantiene la alineación del eje del cuerpo y la cabeza. En casos de sospecha de mielopatía, el monitoreo del potencial evocado motor debe realizarse antes y después del posicionamiento para confirmar la integridad de las vías neurales[29]. En ausencia de tal monitoreo, tam- bién se puede hacer pronación despierta[30]. La parálisis del nervio laríngeo recurrente se ha asociado con procedimientos que involucran el abordaje anterior de columna cervical inferior. La compresión mediada por el tubo endotraqueal del nervio laríngeo recurrente debido a la retracción de la cirugía de la columna cervical anterior es una causa importante de parálisis cordal. Se recomienda controlar con frecuencia la presión del manguito endotraqueal y mantenerlo dentro del rango normal durante el procedimiento. Desinflar el manguito del tubo endotraqueal y volver a inflar solo para sellar la presión después de la colocación de los retractores ayuda a reducir la incidencia de parálisis del nervio laríngeo recurrente[31].

En los procedimientos de la columna lumbar, torácica inferior y sacra, no se requiere el uso de dispositivos de fijación de la cabeza, y la cabeza se coloca sobre un reposacabezas de gel o espuma. Las extremidades superiores generalmente se colocan hacia arriba: posición de Superman para procedimientos en la columna lumbar o por el lado para casos torácicos. Se debe tener cuidado de que el plexo braquial no se estire.

\section{Problemas de la posición prono (Figura 5.2)}

1- Aumento de la presión intraabdominal en la posición prona: Con una posición inadecuada y en pacientes obesos, la presión intraabdominal aumenta con la posición prona que conduce al síndrome compartamental abdominal (SCA) y aumenta el sangrado en los procedimientos de la columna vertebral. Un aumento en la presión intraabdominal de más de 12 $\mathrm{mmHg}$ desde la posición supina es un alto riesgo de desarrollar SCA. Los pacientes con cirugía abdominal previa tienen más riesgo de un SCA. La elevación prolongada de las presiones intraabdominales puede conducir a una disminución de la presión de perfusión que predispone a un fallo multiorgánico. Se debe considerar la identificación de pacientes que están en riesgo de desarrollar una presión intraabdominal alta y monitorear la presión intraabdominal en ellos. Cualquier disminución en la presión arterial, diuresis o el aumento de $\mathrm{PCO}_{2}$ puede apuntar hacia una falla multiorgánica. (32)

2- Parálisis Nerviosa / Neuropraxia: En la posición prona, existe un mayor riesgo de lesión en la columna cervical y el plexo braquial. Las alteraciones de las transmisiones axoplasmáticas debidas al aumento de la presión venosa intraneural, el edema local son los mecanismos fisiopatológicos que conducen a las parálisis de los nervios y las neuropraxias. Esto puede deberse a estiramiento, compresión, isquemia generali- 


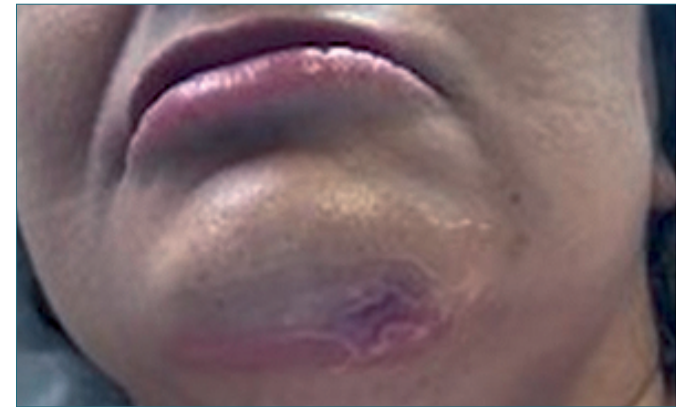

Figuras 5.1 y 5.2 .

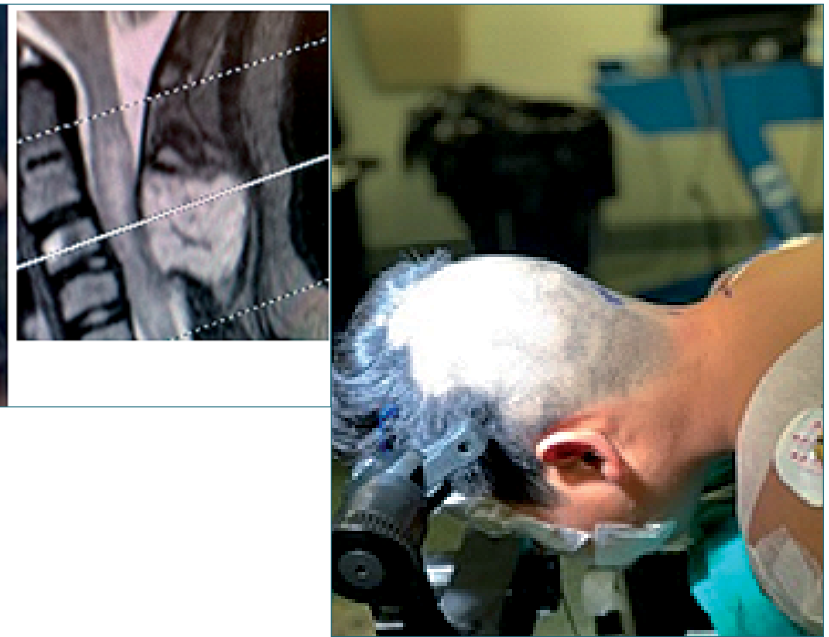

zada o causas metabólicas. Se han identificado varios factores de riesgo, como el sexo masculino, individuos extremadamente delgados, obesos, hipotensión, diabetes mellitus, hipotermia, variaciones anatómicas y desnutrición[33],[34]. La colocación del húmero anterior al tronco y la colocación de acolchado de apoyo para el brazo es beneficiosa para prevenir la lesión del plexo braquial. La palpación del tendón del músculo pectoral mayor se puede usar para controlar la tensión en el plexo braquial[33],[34].

3- Úlceras por presión: En la posición prona, se ejerce una presión extrema sobre el mentón, la frente, reborde supraorbitario, la eminencia malar y las orejas. En promedio, la presión en la cara es de $30 \mathrm{mmHg}$, que está por encima de la presión de perfusión capilar. Puede ser incluso de hasta 50 mmHg[35]. En el tórax las pacientes con senos más grandes son más susceptibles a las lesiones. La rotura mamaria y la necrosis son posibles en las personas con implantes[36]. Se han notificado casos de hematoma, condritis, isquemia y necrosis en el oído. Todos los puntos de presión deben rellenarse con el acolchado adecuado, como almohadas de gel (Figura 5.1).

4- Edema de la cara: Se han observado hinchazón orofaríngea, macroglosia y hematoma sublingual en pacientes en posición prona. El doblado y el estiramiento de los conductos salivales, los vasos sanguíneos y los linfáticos cuando se flexiona el cuello predisponen a inflamaciones de las glándulas salivales[37]. La dislocación de la articulación temporomandibular durante la intubación, la higiene oral deficiente, la deshidratación, la desnutrición, la laringoscopia repetida, el uso de inhibidores de la enzima convertidora de angiotensina son conocidos factores de riesgo para inflamaciones de las glándulas salivales. El uso de bloqueo de mordida suave para evitar la compresión de la lengua es preferible a las vías respiratorias orales duras. El paciente debe ser monitoreado en las primeras horas para detectar estridor de postextubación. 5- Desplazamiento del tubo endotraqueal: LoS pacientes en posición prona tienen un mayor riesgo de desplazamiento del tubo endotraqueal debido al peso de los circuitos respiratorios. La máscara laríngea se puede utilizar de rescate en caso de extubación accidental[13]. Para evitar el desplazamiento es esencial mantener las presiones más altas del manguito.

6- Pérdida de visión perioperatoria: La incidencia de pérdida de la visión perioperatoria después de los procedimientos de la columna vertebral es de 0,05 a 1\%[38]. El posicionamiento defectuoso representa solo un pequeño porcentaje de la pérdida de visión postoperatoria. La presión directa sobre el ojo causa un traumatismo que resulta en edema conjuntival, hemorragia, quemosis y dolor.

7- Neuropatía óptica isquémica: El aumento de la presión intraocular y el aumento de la presión venosa orbital conducen a una disminución de la presión de perfusión en la cabeza del nervio óptico. La neuropatía óptica isquémica posterior es más común después de la operación y es más severa que la anterior[39]. Se sabe que ocurren incluso con suficiente apoyo para el relleno facial. Los factores de riesgo son: tiempo operatorio prolongado, anemia, hipotensión y transfusión sanguínea[40].

8- Oclusión de la arteria central de la retina (síndrome del reposacabezas): Esto puede deberse a la presión directa o indirecta y se caracteriza por un edema periorbital, escleral y una mancha roja en el 
fondo del ojo. El riesgo de oclusión de la arteria central de la retina generalmente se debe a una colocación incorrecta durante la cirugía[40]. La patogenia puede deberse a vasoespasmo, émbolos, compresión o hipotensión[38]. El desprendimiento de placas de la arteria carótida puede causar OACR. La presión directa del reposacabezas puede ser mayor en pacientes con exoftalmos o puente nasal bajo.

9- Ceguera cortical: Esto se debe a un infarto de la arteria cerebral posterior por hipotensión significativa. Esto puede recuperarse lentamente pero no completamente. Los factores de riesgo para la ceguera cortical incluyen paro cardíaco, embolia aérea, hipotensión significativa o hipoxia prolongada. Se encontró que la posición prona y la obesidad se asociaban más comúnmente con el desarrollo de la ceguera cortical[40].

10- Glaucoma de cierre de ángulo agudo: La posición prona desplaza el diafragma del iris de la lente hacia adelante y obstruye el flujo del humor acuoso. Esto provoca un aumento repentino de la presión intraocular. En pacientes de alto riesgo, incluso los procedimientos quirúrgicos cortos pueden precipitar el glaucoma de cierre de ángulo agudo. La se han asociado con la duración del procedimiento durante más de $6 \mathrm{~h}$, pérdida de sangre de más de $1.000 \mathrm{ml}$, DM, hipertensión. ION se produce independientemente del marco y la frecuencia de los controles oculares. Se puede prevenir evitando cambios de presión arterial, manteniendo un $M A P \geq 70 \mathrm{mmHg}$, hematocrito> $25 \mathrm{mmHg}$.

\section{f) Posición de concorde}

Se prefiere esta posición para el abordaje suboc- cipital lateral para los tumores de la fosa posterior, especialmente para el abordaje occipital transtentorial y supracerebeloso infratentorial. El paciente está posicionado como para una posición suboccipital en la línea media. La cabeza está asegurada con un fijador antes de voltearse a prono. Luego se flexiona con la región toracolumbar extendida de modo que la cabeza se eleva sobre el corazón. Se modifica ligeramente para hacer que el sitio de la craneotomía o la craniectomía sea más prominente al girar la cabeza del paciente aproximadamente $45^{\circ}$ hacia el hombro ipsilateral a la lesión. El posicionamiento de Trendelenburg inverso se realiza para mejorar el retorno venoso (Figura 7). Hay una menor incidencia de embolias aéreas[15] y menos fatiga para el cirujano. Además, no se necesita instrumentación complicada. Las complicaciones son las mismas que las de la posición prona[16].

\section{g) Tres cuartos prono (lateral oblicuo o semipro- no)}

La posición tres cuartos prono (Figura 8) es muy similar a la posición lateral y se usa para acercarse tanto a la fosa posterior como a la región parietooccipital. La cabeza se coloca en un fijador y el brazo no dependiente se coloca detrás del cuerpo en posición de dormir. Sin embargo, si se usa un abordaje suboccipital, la mano puede estar sujeta con cinta adhesiva hacia el pie. La parte superior del hombro puede separarse del área de la cabeza y el cuello hacia el pie de la cama con una cinta. El tronco del paciente está apoyado por correas, cintas o tirantes. La extremidad inferior dependiente se extiende con la rodilla acol-

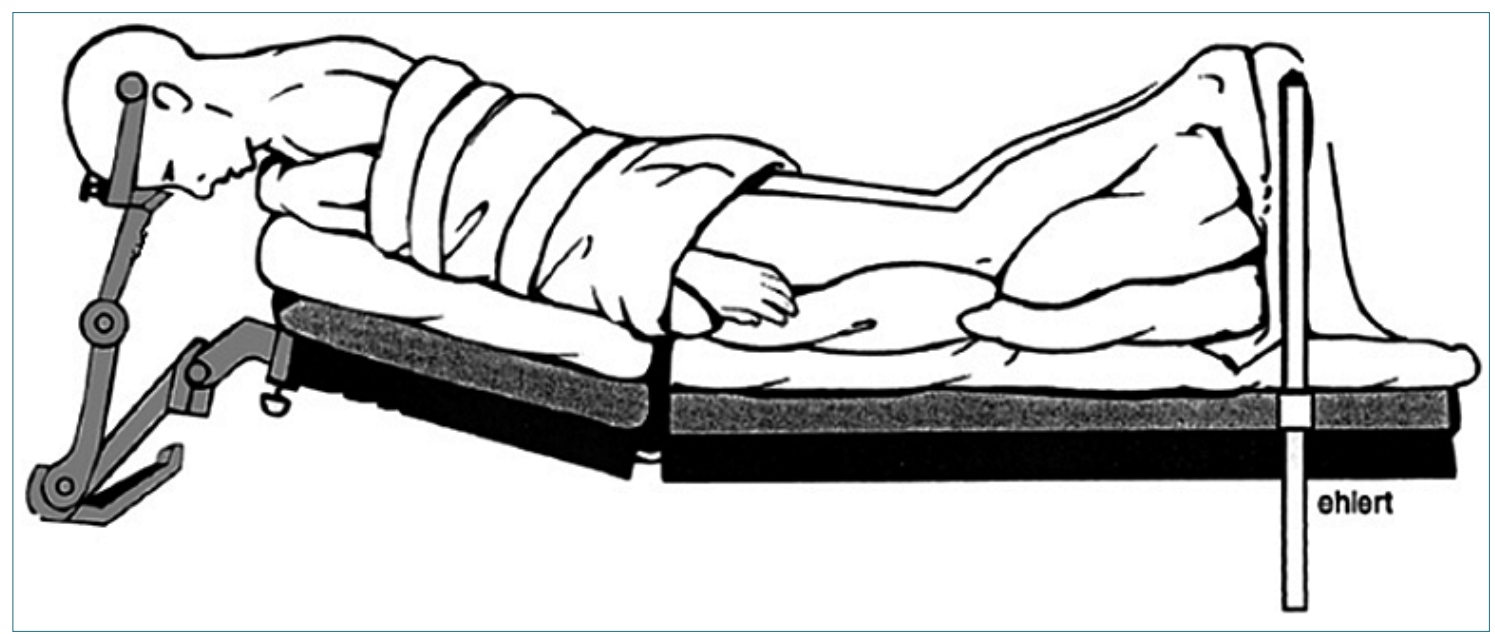

Figura 7. Posición concorde. Imagen tomada de: Rozet I, Vavilala MS. Risks and benefits of patient positioning during neurosurgical care. Anesthesiol Clin September 2007;25(3):631-53. 


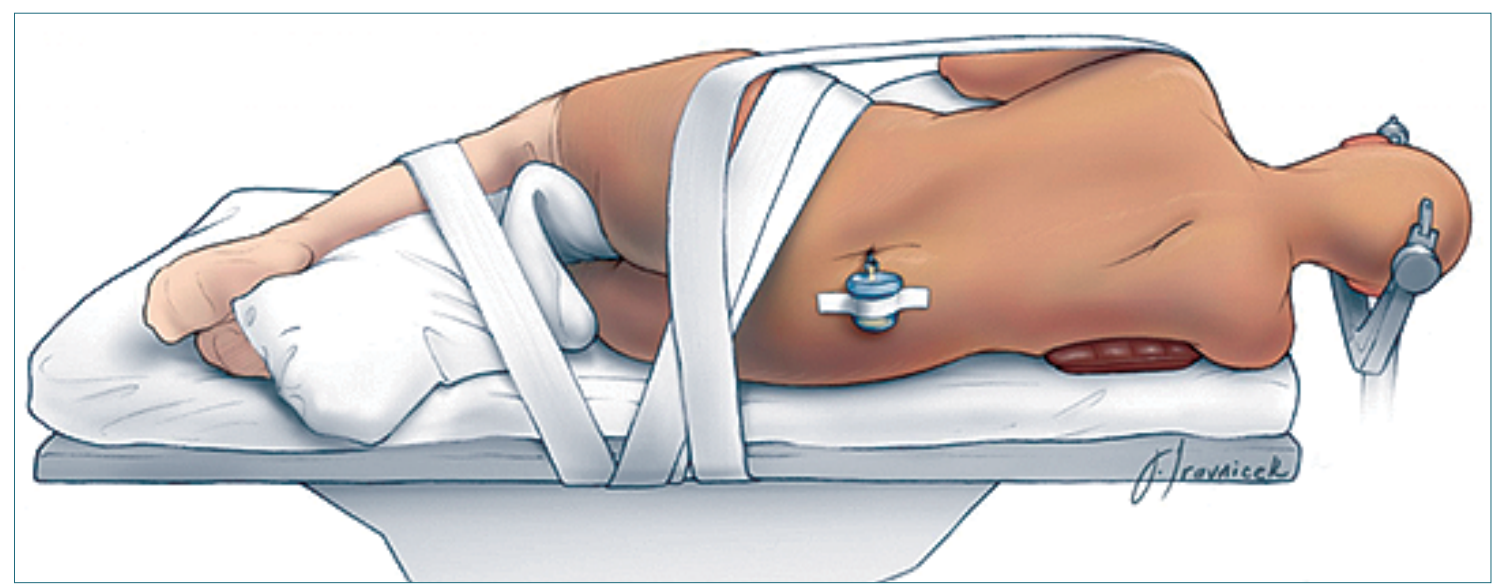

Figura 8. Posición semiprono. Image tomada de : https://www.neurosurgicalatlas.com/volumes/principles-of-cranial-surgery/ patient-positioning.

chada para evitar la presión sobre el nervio peroneo, y la extremidad inferior superior está flexionada en la cadera y la rodilla. Las ventajas son que es cómodo para el cirujano y disminuye el riesgo de embolia aérea venosa (EAV).

\section{h) Abordaje para la cirugía transnasal transesfe- noidal}

El paciente suele colocarse en posición supina con la cabeza sobre un reposacabezas de herradura y las manos a los lados. La cabeza se gira sutilmente hacia la posición del cirujano que realizara la endoscopia. La posición de Trendelenburg inversa se realiza para disminuir la congestión venosa. La colocación incorrecta de los pacientes para el abordaje transesfenoidal hace que la cirugía sea difícil y peligrosa, ya que una pequeña alteración en la trayectoria planificada puede tener consecuencias desastrosas como la lesión de la carótida o el tronco cerebral[17] (Figura 9).

\section{i) Posición sentada}

La posición sentada ofrece un acceso óptimo a las lesiones de la línea media. También mejora el drenaje venoso cerebral, disminuyendo la PIC, disminuye la necesidad de retracción cerebelosa, promueve el drenaje de LCR por medio de la gravedad, lo que permite un campo quirúrgico limpio, la visualización de puntos de sangrado y visión de la cara del paciente sin obstrucciones, lo que permite observar las respuestas motoras de los nervios craneales al estímulo.

Si bien la posición sentada ofrece distintas ventajas para el cirujano, la extensión de la extirpación del tumor, los resultados neurológicos y la preservación del nervio facial no han demostrado ser muy diferentes en comparación con la posición lateral[18]. Las cirugías cervicales instrumentadas también han sido hechas en esta posición.

En la posición sentada, hay una acumulación venosa significativa de las extremidades inferiores debido al efecto de la gravedad que conduce a la dismi-

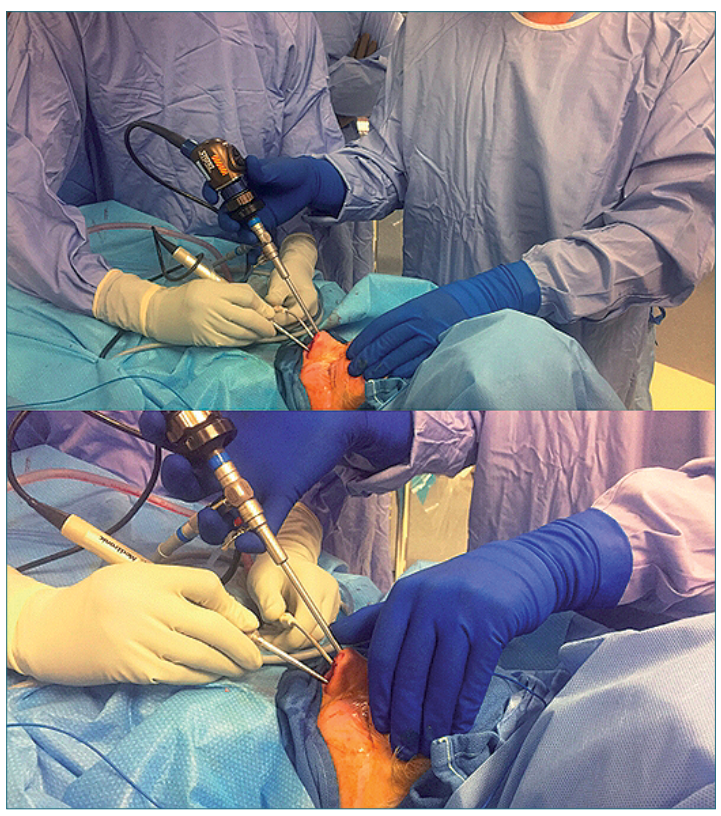

Figura 9: Posición para cirugía transesfenoidal. Image tomada de : https://www.neurosurgicalatlas.com/volumes/ principles-of-cranial-surgery/patient-positioning. 
nución del GC y, por lo tanto, de hipotensión arterial. La frecuencia cardíaca y la RVS se incrementan. VS e IC están disminuidos.

Se han observado aumentos en la capacidad funcional residual (CFR) y el volumen pulmonar total (VPT). Sin embargo, como la perfusión es limitada, no se observa ningún beneficio importante en la oxigenación. La ventilación no está impedida a medida que se mejora el movimiento diafragmático, lo que reduce la presión sobre la vía respiratoria.

Dado que la presión arterial se reduce en 0,77 $\mathrm{mmHg}$ por cada gradiente centimétrico sobre el corazón, la PPC se reduce después de colocar al paciente en posición sentada, lo que conduce al posible desarrollo de isquemia cerebral. La PIC también se reduce en la posición sentada. Una posición semisentada que apunta a crear una presión positiva en los senos transversales y sigmoidales, con la cabeza más baja y las piernas más altas colocadas por encima de la parte superior de la cabeza, disminuye la incidencia y la gravedad de EAV[19].

El paciente se coloca inicialmente en posición supina sobre la mesa de operaciones y se lo anestesia. La cabeza se coloca en el fijador. Luego se coloca en posición sentada elevando lentamente la parte posterior de la mesa de operaciones gradualmente durante minutos, manteniendo la estabilidad hemodinámica. Es importante evitar la hiperventilación, ya que puede comprometer el flujo sanguíneo cerebral[20].

Cuando el paciente se coloca en posición sentado, la mesa de operaciones se flexiona, se elevan los muslos y se deja caer el pie por fuera la mesa, flexionando las rodillas para evitar estirar el nervio ciático. Se puede colocar una almohada debajo de las rodillas. La mesa de operaciones se inclina hacia atrás cuando la mesa se flexiona. El cuello del paciente se mantiene en posición neutra o flexionada. Los brazos deben estar asegurados a través del cuerpo o un reposabrazos para evitar que el hombro caiga, estirando hacia abajo el plexo braquial. No se debe permitir que los pies cuelguen de la mesa y los tobillos deben apoyarse para evitar lesiones en el tendón de Aquiles[1] (Figura 10).

Para evitar lesiones durante la flexión del cuello, es preferible monitorear el potencial evocado somatosensorial (PESS) mientras se coloca. Se recomienda que el paciente sea revisado antes de la operación para determinar el grado de flexión posible del cuello. Los cambios hemodinámicos durante el posicionamiento pueden verse influenciados por factores del paciente como el estado del volumen intravascular, la hipotensión preexistente y el IMC. Posicionar al paciente con la flexión de las caderas y elevar las rodillas

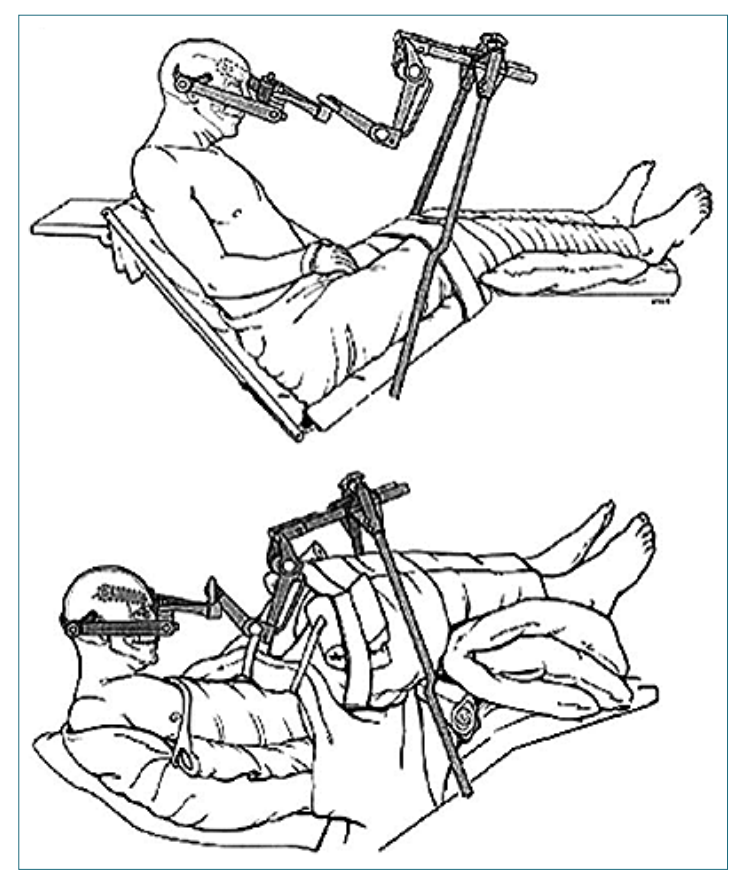

Figura 10. Posición sentada. Imagen tomada de: Rozet I, Vavilala MS. Risks and benefits of patient positioning during neurosurgical care. Anesthesiol Clin September 2007;25(3):631-53.

al nivel del corazón ayuda a minimizar la hipotensión. El posicionamiento de fase lenta después de una hidratación adecuada durante un período de 5 a 10 minutos con bolos de vasopresores intermitentes, según sea necesario, evitará una caída brusca de la presión arterial. Al envolver las extremidades inferiores, también promueve el retorno venoso desde las extremidades inferiores.

\section{Complicaciones asociadas con la posición sentada}

\section{a) Embolia aérea venosa (EAV)}

Un estudio con Doppler precordial,demostró que la incidencia varía entre $25 \%$ y $50 \%$ [21] y en $76 \%$ con eco Doppler transesofágico (ETE)[22]. La presión venosa negativa y la exposición de los senos venosos óseos al aire son los responsables de la EAV. Se han descrito varias modalidades de monitoreo con diferentes sensibilidades, como la capnografía, la excreción fraccional de nitrógeno, el eco Doppler transtorácico (ETT), el catéter derecho del corazón, el ETE y la medición de oxígeno transcutáneo. La EAV reduce el GC al provocar un bloqueo de aire y la posterior sobredistensión mecánica del ventrículo derecho y la obstrucción de la circulación pulmonar. El ETT es el 
mejor dispositivo no invasivo para monitorizar la EAV.

La irrigación del campo con dióxido de carbono se ha utilizado para reducir los efectos hemodinámicos del EAV[23]. El catéter con balón intrajugular también se usa para prevenir el EAV[24]. Se ha demostrado que el abordaje paramediano conduce a un aumento de la incidencia de la EAV en comparación con el abordaje mediano[25].

\section{b) Embolia aérea paradójica}

El gradiente entre las aurículas es un factor importante en la fisiopatología de la EAV. Cualquier condición que aumente la presión auricular derecha en relación con la presión auricular izquierda puede predisponer a una embolia aérea paradójica (EAP). La aplicación y liberación de presión positiva al final de la espiración aumenta las presiones de la aurícula derecha, lo que favorece la derivación de derecha a izquierda y la EAP. Los pacientes con riesgo de EAP, como aquellos con derivaciones de derecha a izquierda, el foramen oval permeable debe identificarse previo a la operación.

\section{c) Normocéfalo}

La incidencia de normocéfalo es casi del $100 \%$. Disminución del volumen cerebral secundario a la administración de manitol, hiperventilación, drenaje intraoperatorio de LCR predisponente al normocéfa- lo. Evitando la deshidratación intracraneal, la hiperventilación durante el cierre de la duramadre para facilitar la expansión del cerebro ayuda a disminuir la incidencia de neumocefalia. La confusión, el dolor de cabeza, las convulsiones y los déficits neurológicos en el postoperatorio inmediato deben evaluarse con una tomografía computarizada rápida. La rápida evacuación de la tensión neumocefalia da como resultado una pronta recuperación.

\section{d) Cuadriplejia}

La flexión extrema del cuello estira la médula en C5, lo que conduce a una disminución de la perfusión regional medular especialmente durante los períodos de hipotensión. Los PESS se puede usar para monitorear la adecuada perfusión regional medular.

\section{e) Lesiones nerviosas}

La neuropatía peronea común a menudo ocurre secundaria a la compresión del nervio a nivel del cuello del peroné. La hiperflexión del muslo puede provocar un estiramiento de la división peroneal del nervio ciático. La lesión del nervio laríngeo recurrente también se puede presentar secundaria a la flexión extrema.

\section{f) Otras complicaciones}

Macroglosia puede ocurrir y conducir a la obs-

\begin{tabular}{|c|c|c|c|}
\hline Abordaje & Ubicación de la lesión & Posición del cuerpo & Posición de la cabeza \\
\hline Pterional & $\begin{array}{l}\text { Fosa craneana anterior y media, } \\
\text { aneurisma de circulación ante- } \\
\text { rior }\end{array}$ & Supino & $\begin{array}{l}\text { Flexión, rotación de la cabeza } \\
\text { hasta } 45^{\circ} \text { y elevación de la ca- } \\
\text { beza. Cigoma prominente }\end{array}$ \\
\hline Temporal y subtemporal & $\begin{array}{l}\text { Apex petroso, aneurisma del } \\
\text { top de la basilar, fosa craneana } \\
\text { media }\end{array}$ & Supino, lateral & $\begin{array}{l}\text { Flexión, rotación en } 90^{\circ} \text {, gel en } \\
\text { hombro ipsilateral a la lesión }\end{array}$ \\
\hline Anterior parasagital & $\begin{array}{l}\text { Aneurismas distales de circula- } \\
\text { ción anterior. Tumores de los } \\
\text { ventrículos laterales y tercer } \\
\text { ventrículo, abordaje interhemis- } \\
\text { férico }\end{array}$ & Supino & $\begin{array}{l}\text { Neutral en lineal media, flexión } \\
\text { variable }\end{array}$ \\
\hline Subfrontal & Fosa craneana anterior & Supino & Neutral, extensión variable. \\
\hline Posterior parasagital & Lesiones región pineal & Supino, oblicuo, 3/4 prono. & Flexión variable \\
\hline Suboccipital medial & $\begin{array}{l}4^{\circ} \text { ventrículo, cerebelo línea } \\
\text { media, región pineal }\end{array}$ & Prono, concorde, Park bench & Flexión variable \\
\hline Suboccipital lateral & $\begin{array}{l}\text { Angulo ponto cerebeloso, cere- } \\
\text { beloso lateral }\end{array}$ & $\begin{array}{l}\text { Concorde, prono, lateral, } \\
\text { park bench, sentado }\end{array}$ & Flexión variable \\
\hline Transesfenoidal & $\begin{array}{l}\text { Silla turca, fosa anterior, supra- } \\
\text { selar, paraselar }\end{array}$ & Supino & $\begin{array}{l}\text { Rotada sutilmente, puente na- } \\
\text { sal en } 45^{\circ}\end{array}$ \\
\hline
\end{tabular}


trucción de la vía aérea. Esto es más pronunciado en los pacientes pediátricos donde hay una laringe anterior, un diámetro traqueal pequeño y una lengua grande. La flexión sostenida del cuello, la duración prolongada y el uso de las vías respiratorias orales se han implicado en la patogénesis de la macroglosia. Los bloqueos de mordida y las sondas de diámetro más pequeño minimizarán la incidencia de macroglosia (Tabla 4).

\section{Conclusiones}

Los procedimientos quirúrgicos del cerebro y la co- lumna vertebral requieren una posición compleja para que el cirujano pueda acceder fácilmente al objetivo quirúrgico. Requiere un equilibrio entre el mejor abordaje quirúrgico y la posición fisiológicamente óptima para el paciente. Dentro de los límites fisiológicos, la posición debe modificarse para ayudar a los objetivos quirúrgicos, como la reducción del sangrado, la PIC y la retracción cerebral mínima. Se debe ofrecer atención cuidadosa y una protección meticulosa a los tejidos vulnerables, especialmente durante la duración prolongada de la cirugía. El posicionamiento óptimo es la clave para prevenir la morbilidad y mejorar los resultados quirúrgicos después de procedimientos neuroquirúrgicos complejos.

\section{Referencias}

1. Richard Winn H. General principles of operative positioning. In: YOUMANS neurological surgery. 5th ed. Philadelphia, PA: Saunders, Elsevier; 2004. p. 595-621.

2. Mitchell J. Vertebral artery blood flow velocity changes associated with cervical spine rotation: a meta-analysis of the evidence with implications for professional practice. J Man Manip Ther 2009;17(1):46-57. https://doi./10 .1179/106698109790818160

3. Sakaguchi M, Kitagawa K, Hougaku $\mathrm{H}$, Hashimoto $\mathrm{H}$, Nagai $\mathrm{Y}$, Yamagami $\mathrm{H}$, et al. Mechanical compression of the extracranial vertebral artery during neck rotation. Neurology September 23, 2003;61(6):8457. https://doi.org/10.1212/01. WNL.0000078081.12097.AE

4. Brambrink AM, Kirsch JR. Positioning the patient for neurosurgical operations essentials of neurosurgical anesthesia \& critical care. In: Essentials of neurosurgical anesthesia \& critical care. New York: Springer; 2012. p. 151-8. https://doi. org/10.1007/978-0-387-09562-2

5. Haure P, Cold GE, Hansen TM, Larsen JR. The ICP-Lowering effect of $10^{\circ}$ reverse Trendelenburg position during craniotomy is stable during a 10-minute period. J Neurosurg Anaesthesiol October 2003;15(4):297-301. https:// doi.org/10.1097/00008506200310000-00001

6. Tankisi A, Rolighed Larsen J, Rasmussen M, Dahl B, Cold GE. The effects of 10 degrees reverse trendelenburg position on ICP and CPP in prone positioned patients subjected to craniotomy for occipital or cerebellar tumours. Acta Neurochir (Wien) July 2002;144(7):665-70. https:// doi.org/10.1007/s00701-0020957-y

7. Tankisi A, Cold GE. Optimal reverse trendelenburg position in patients undergoing craniotomy for cerebral tumors. J Neurosurg February 2007;106(2):23944. https://doi.org/10.3171/ jns.2007.106.2.239

8. Rozet I, Vavilala MS. Risks and benefits of patient positioning during neurosurgical care. Anesthesiol Clin September 2007;25(3):631-53. x. https://doi.org/10.1016/j. anclin.2007.05.009

9. Tew Jr JM, Scodary DJ. Basic techniques and surgical positioning. In: Brain Surgery, Complication avoidance and management. New York: Churchill Livingston Inc.; 1993. p. 31-51.

10. Manikandan S, Umamaheswara Rao GS. Effect of surgical position on pulmonary gas exchange in neurosurgical patients. Indian J Anaesth 2002;46(5):356-9.

11. Winfree CJ, Kline DG. Intraoperative positioning nerve injuries. Surg Neurol January 2005;63(1):5-18. discussion 18. https://doi.org/10.1016/j. surneu.2004.03.024

12. Schubert A. Positioning injuries in anesthesia: an update. Adv Anesth October 1, 2008;26:3165. https://doi.org/10.1016/j. aan.2008.07.009

13. Raphael J, Rosenthal-Ganon T, Gozal Y. Emergency airway management with a laryngeal mask airway in a patient placed in the prone position. J Clin Anesth November 2004;16(7):560-1. https://doi.org/10.1016/j.jclinane.2004.03.004

14. Edgcombe H, Carter K, Yarrow $\mathrm{S}$. Anaesthesia in the prone position. Br J Anaesth November 23, 2007;100(2):165-83. https:// doi.org/10.1093/bja/aem380

15. Albin MS, Carroll RG, Maroon JC. Clinical considerations 
concerning detection of venous air embolism. Neurosurgery December 1978;3(3):380-4. https:// doi.org/10.1227/00006123197811000-00009

16. Kobayashi S, Sugita K, Tanaka Y, Kyoshima K. Infratentorial approach to the pineal region in the prone position: concorde position. Technical note. J Neurosurg January 1983;58(1):1413. https://doi.org/10.3171/ jns.1983.58.1.0141

17. Crowley RW, Dumont AS, McKisic MS, Jane Sr JA. Positioning for cranial surgery. In: YOUMANS neurological surgery. 6th ed. Philadelphia, PA: Saunders, Elsevier; 2011. p. 442-6. https:// doi.org/10.1016/B978-1-41605316-3.00027-7

18. Turgut N, Ali A, Turkmen A, Altan A, Bikmaz K, Iplikcioglu AC. Comparison of surgical approaches in neurosurgical patients experiencing venous air embolism in the sitting position. West Indian Med J August 2014;63(4):388. https://doi. org/10.7727/wimj.2013.133

19. Gracia I, Fabregas N. Craniotomy in sitting position: anesthesiology management. Curr Opin Anaesthesiol October 2014;27(5):47483. https://doi.org/10.1097/ ACO.0000000000000104

20. Tindall GT, Craddock A, Greenfield JC. Effects of the sitting position on blood flow in the internal carotid artery of man during general anesthesia. J Neurosurg April 1967;26(4):3839. https://doi.org/10.3171/ jns.1967.26.4.0383

21. Voorhies RM, Fraser RA, Van Poznak A. Prevention of air embolism with positive end expiratory pressure. Neurosurgery May 1983;12(5):503-6. https:// doi.org/10.1227/00006123198305000-00004

22. Papadopoulos G, Kuhly P, Brock
M, Rudolph KH, Link J, Eyrich K. Venous and paradoxical air embolism in the sitting position. A prospective study with transoesophageal echocardiography. Acta Neurochir (Wien) 1994;126(2-4):140-3. https://doi. org/10.1007/BF01476424

23. Longatti $P$, Marton $E$, Feletti $A$, Falzarano M, Canova G, Sorbara C. Carbon dioxide field flooding reduces the hemodynamic effects of venous air embolism occurring in the sitting position. Childs Nerv Syst August 2015;31(8):1321-6. https://doi. org/10.1007/s00381-015-27422

24. Eckle VS, Neumann B, Greiner TO, Wendel HP, Grasshoff C. Intrajugular balloon catheter reduces air embolism in vitro and in vivo. $\mathrm{Br} J$ Anaesth June 2015;114(6):973-8. https://doi. org/10.1093/bja/aev040

25. Turgut N, Ali A, Turkmen A, Altan A, Bikmaz K, Iplikcioglu AC. Comparison of surgical approaches in neurosurgical patients experiencing venous air embolism in the sitting position. West Indian Med J August 2014;63(4):388. https://doi. org/10.7727/wimj.2013.133

26. Palmon SC, Kirsch JR, Depper JA, Toung TJ. The effect of the prone position on pulmonary mechanics is frame-dependent. Anesth Analg November 1998;87(5):1175-80. https:// doi.org/10.1097/00000539199811000-00037

27. Pearce DJ. The role of posture in laminectomy. Proc R Soc Med February 1957;50(2):109-12. https:// doi.org/10.1097/00132586195712000-00014

28. Angevine PD, Gigante PR. Patient positioning for spinal surgery. In: YOUMANS neurological surgery. 6th ed. Philadelphia, PA: Saunders, Elsevier; 2011. p.
447-52. https://doi.org/10.1016/ B978-1-4160-5316-3.00028-9

29. Ofiram E, Lonstein JE, Skinner S, Perra JH. "The disappearing evoked potentials": a special problem of positioning patients with skeletal dysplasia: case report. Spine June 15, 2006;31(14):E464-70. https://doi.org/10.1097/01. brs.0000222122.37415.4d

30. Douglass J, Fraser J, Andrzejowski J. Awake intubation and awake prone positioning of a morbidly obese patient for lumbar spine surgery. Anaesthesia February 2014;69(2):1669. https://doi.org/10.1111/ anae. 12387

31. Tan TP, Govindarajulu AP, Massicotte EM, Venkatraghavan L. Vocal cord palsy after anterior cervical spine surgery: a qualitative systematic review. Spine July 1, 2014;14(7):133242. https://doi.org/10.1016/j. spinee.2014.02.017

32. Shih P, Slimack NP, Roy A, Fessler RG, Koski TR. Abdominal complications following posterior spinal fusion in patients with previous abdominal surgeries. Neurosurg Focus October 2011;31(4):E16. https://doi.g/10. 3171/2011.8.FOCUS11132

33. Akhavan A, Gainsburg DM, Stock JA. Complications associated with patient positioning in urologic surgery. Urology December 2010;76(6):1309-16 https://doi.org/10.1016/j.urology.2010.02.060

34. Mobley SR, Miller BT, Astor FC, Fine B, Halliday NJ. Prone positioning for head and neck reconstructive surgery. Head Neck November 2007;29(11):10415. https://doi.org/10.1002/ hed. 20650

35. Leibovitch I, Casson R, Laforest C, Selva D. Ischemic orbital compartment syndrome as a complication of spinal surgery in 
the prone position. Ophthalmology January 2006;113(1):1058. https://doi.org/10.1016/j. ophtha.2005.09.025

36. Mobley SR, Miller BT, Astor FC, Fine B, Halliday NJ. Prone positioning for head and neck reconstructive surgery. Head Neck November 2007;29(11):1041-5. https://doi. org/10.1002/hed.20650

37. Hans $P$, Demoitié J, Collignon L, Bex V, Bonhomme V. Acute bilateral submandibular swelling following surgery in prone position. Eur J Anaesthesiol January 2006;23(1):834. https://doi.org/10.1017/ S0265021505231824

38. Stambough JL, Dolan D, Werner R, Godfrey E. Ophthalmologic complications associated with prone positioning in spine surgery. J Am Acad Orthop Surg March 2007;15(3):156-65. https:// doi.org/10.5435/00124635200703000-00005

39. Lee LA, Newman NJ, Wagner
TA, Dettori JR, Dettori NJ. Postoperative ischemic optic neuropathy. Spine April 20, 2010;35(Suppl. 9): S10516. https://doi.org/10.1097/ BRS.0b013e3181d8344d

40. Li A, Swinney C, Veeravagu A, Bhatti I, Ratliff J. Postoperative visual loss following lumbar spine surgery: a review of risk factors by diagnosis. World Neurosurg December 2015;84(6):201021. https://doi.org/10.1016/j. wneu.2015.08.030 